\title{
Application of surveying and mapping technology in airport construction
}

\author{
Lan Cheng ${ }^{1,2}$, Fengchen Chen ${ }^{1,2, *}$, Ke Tang ${ }^{1,2,3}$, Wenjia Du ${ }^{1,2}$ \\ ${ }^{1}$ China Airport Construction Group Co., Ltd., Beijing, China \\ ${ }^{2}$ Beijing Super-Creative Airport Technical Ltd., Beijing, China \\ ${ }^{3}$ School of Automation Science and Electrical Engineering, Beihang University, Beijing, China
}

\begin{abstract}
Due to the accelerating urbanization process, land resources are tight, the construction environment becomes more and more complex and severe. In particular, the future airport will be built on the mountain or be constructed reclamation airport, the terrain and geological conditions of the airport construction are complex and the climate is harsh. These all raise higher requirements for the accuracy and safety of airport planning, design and construction. This paper combines the mapping technology with the key links of airport construction, and elaborates on the key technologies, which provides a good reference for related projects and is of great significance for improving the efficiency and quality of airport construction.
\end{abstract}

\section{Introduction}

The rapid development of science and technology has promoted the advancement of theory, technology and methods in various fields. With the continuous development of computer technology, communication technology and geographic information technology, the information collection methods in the field of surveying and mapping are more and more intelligent and digital, and the data processing speed is more and more fast, the accuracy of surveying and mapping results is getting higher and higher, and more real-time and visual [1]. The application of surveying and mapping technology to airport construction will greatly improve the technical level of airport construction and provide fast and reliable data support for airport construction [2].

In the early stage of site selection, in order to select the best runway orientation, it is necessary to consider such issues as airport meteorology, height of surrounding obstacles and clearance limited plane. The traditional manual site selection method relies on the primary selection and site reconnaissance by experienced designers, and estimates the clearance limited plane. It is very difficult to select the site in mountainous airports, reclamation airports and other harsh environments. The traditional estimation of cubic meter of earth and stone volume and the calculation of cubic meter of earth and stone volume after the completion of construction are often analyzed and calculated by airport designers and managers using manual map method. Due to the large amount of engineering, the calculation is complicated and the accuracy is difficult to ensure. This paper puts forward a new idea for airport construction, which is to apply surveying and mapping technology to airport construction, so as to improve the technical level of airport construction [3].

\section{Surveying and mapping technology mainly used in airport construction}

In the scene of airport construction, various surveying and mapping technologies are organically combined and multi-source data fusion technology is used to obtain more comprehensive and accurate information.

\subsection{Global navigation satellite system (GNSS)}

GNSS is a positioning system that uses radio signals loaded with special positioning information to realize positioning measurement by continuously broadcasting a certain frequency to the ground. GNSS generally consists of three parts:

(1) A constellation of satellites operating in space. The constellation system composed of several satellites sends time signal ranging signal and instantaneous coordinate position signal to the ground.

(2) Ground control. By receiving the above signals, it can accurately determine the satellite's orbit coordinates and clock differences, monitor whether its operation is normal, and inject new satellite orbit coordinates into the satellite to carry out necessary satellite orbit correction and adjustment control [4].

(3) User section. It receives various signals sent by satellite broadcasting through the user's satellite signal receiver and processes and calculates them to determine the user's final position. The user receiver is usually fixed to a fixed target on the ground or to a vehicle for the purpose of positioning and navigation.

\footnotetext{
*Corresponding author: 13693228115@ 163.com
} 
Currently, the global satellite navigation and positioning system that is in operation includes the United States' Global Positioning System (GPS), China's Beidou Satellite Navigation System (BDS), the European Union's GALILEO, and Russia's GLONASS [5].

\subsection{Low-altitude photogrammetry}

With the development of Unmanned Aerial Vehicle (UAV) technology, low-altitude airspace is gradually opening, and low-altitude photogrammetry technology has been widely used due to its fast response speed and high precision. The most common way to use lowaltitude photogrammetry is to mount the camera on a UAV and take photos vertically. The specific implementation method is: the UAV flies along the preplanned route, and takes photos on the ground. It is necessary to maintain a fixed heading overlap between adjacent photos, and the overlapping portions constitute a stereo pair. After performing the photo taking task of one route, the UAV enters the adjacent untaken route [6]. There must also be a certain degree of lateral overlap between adjacent route photos, and the overlap of mountainous areas is greater than the overlap of plain areas.

\subsection{Geographic information system (GIS)}

GIS is a system that stores and manages spatial data. It also supports query, retrieval and analysis in the form of graphical and attribute data. The use of GIS can intuitively and closely link various types of data and spatial locations in the airport construction phase, facilitating overlay analysis, obtaining comprehensive analysis results, and visualizing airport information [7].

\section{Integrated application of surveying and mapping technology in airport construction}

There are three main applications of mapping techniques in airport construction: 1) Global Navigation Satellite System (GNSS), 2) Low-altitude photogrammetry, and 3) Geographic Information System (GIS), which are independent of each other, and can interact and superimpose data when needed. GNSS can obtain the target positioning coordinates in an instant, but can not obtain the geographic attributes of the anchor points; low-altitude photogrammetry results can get the scene information well, but can not integrate other layer overlay analysis; GIS can load low-altitude photogrammetry results into the GIS platform, which can support query, retrieval, analysis and comprehensive processing [8]. Low-altitude photogrammetry provides timely, accurate and comprehensive image data for GIS platform due to its current and macroscopic characteristics. At the same time, GNSS also acquires coordinates of arbitrary objects on the image information.
The application of surveying and mapping integration technology runs through the entire process of airport construction. For example, during site selection and preliminary survey, GNSS positioning technology can be used to locate the position and direction of the runway. The low-altitude photogrammetry technology can be used to perform aerial photography on the proposed site to obtain image information. The image and positioning information can be imported into the GIS platform to analyze the feature information of the selected site. In the detailed survey, GNSS technology and national control points can be used to jointly establish a plane control network, and then use GNSS RTK technology combined with the total station to collect terrain data. The terrain data can be imported into the GIS platform [9], and the geological information obtained from the survey can be used to realize the topographic geological visualization of the selected area. In the construction stage, the GNSS RTK technology can be used for the construction stakeout. At the same time, the low-altitude photogrammetry technology can be used to obtain the image and contour line. Comprehensive analysis. The progress profile of each stage of the construction and the specific amount of earth and stone can be obtained. In the operation and maintenance stage of the airport, low-altitude photogrammetry technology can be used to collect the types and quantities of disease on the airport road surface. GNSS positioning technology can be used to locate each disease and import it into the GIS platform to realize the visualization of the road surface disease. At the same time, low-altitude photogrammetry can also be used to obtain 3D data of airport construction. Combined with geographic information system and GNSS positioning technology, the airport design map, clearance obstacle map, airport planning plan, etc. can be positioned in the real scene to be more intuitive and specific. The way to evaluate the rationality and feasibility of the program. Highresolution mapping results ensure that ground resources, meteorological information, airspace resources, and social conditions are clearly visible. A variety of highprecision models that integrate multiple surveying and mapping technologies to promote the development of airport engineering to a higher level of technology.

\section{Application of surveying and mapping technology in airport construction.}

\subsection{Calculation of cubic meter of earth and stone volume}

In the process of airport construction, cubic meter of earth and stone volume is an important factor in the project cost estimate and the selection of airport construction plan, as well as an important content of engineering design. There are two commonly used methods for calculating cubic meter of earth and stone volume, namely, DTM method and grid method.

DTM is the digital terrain model. DTM method can well satisfy the calculation of cubic meter of earth and stone volume in the construction scene of complex 
terrain, and improve the calculation accuracy and efficiency. DTM can be obtained by field acquisition and measurement area image of UAV. In practice, DTM is applied to calculate cubic meter of earth and stone volume. It is necessary to collect the ground information after excavation and filling in the area to be measured, and generate the expression of terrain surface information, morphology and properties of excavation and filling to calculate cubic meter of earth and stone volume.

The grid method is suitable for construction scenes with relatively regular and flat terrain. In practice, the construction area is divided into several squares of 5 20 meters. RTK technology is used to measure the natural elevation of each corner point of the grid. According to the difference between the designed elevation and the natural elevation of each point, the position of zero line is obtained, and then the work quantity of each grid is obtained, and the total amount of cubic meter of earth and stone is obtained by adding.

In practical projects, contour lines are often used to lay the corresponding grid, and the elevation of the vertices of each grid is obtained by contour lines. However, the contour lines cannot completely reflect the topographic conditions, so the method is more suitable for the situation where the topographic conditions are gentle, the ground slope rules, and the construction scope is large.

\subsection{The airport site selection}

At present, the airport site selection still uses the traditional site selection method, the specific steps are as follows:

(1) Make a preliminary selection on the map, and identify a number of sites.

(2) Make an on-the-spot survey, and select 3 4 good sites for further site reconnaissance.

(3) Targeted survey, and select the best site.

Preliminary selection and on-the-spot survey are to preliminarily find out the main conditions of various alternative sites and provide basis for site selection. For sites with basic construction conditions, the location and direction of runway should be preliminarily determined on the site. In the targeted survey, the situation of the remaining 3 4 sites should be comprehensively analyzed, including airport planning and cost estimation, clearance conditions, geological conditions and meteorological conditions.

The disadvantages of traditional site selection mainly include:

(1) The drawings are not realistic, the actual topography is not consistent with the drawings, many artificial obstacles cannot be reflected in the drawings, and the airport planning and design are unreasonable [10].

(2) It takes a lot of time, manpower and material resources to carry out field reconnaissance.

(3) It is difficult to compare multiple schemes by a uniform standard.
Using surveying and mapping technology as core, low-altitude photogrammetry technology is applied to obtain 3D data, contour data and orthophotography images, etc., to provide real high-resolution topography and images of the site selection area of the airport and direct visual effects to site selection personnel. At the same time, designers can make full use of the rich surface information contained in the surveying and mapping results to obtain various geological and clearance data related to airport construction, so as to facilitate the selection of specific plans.

\section{Conclusion}

The application of surveying and mapping technology has greatly improved the technical level of airport construction, raised up new ideas and methods for airport construction, and provided a good technical guarantee for modern airport construction. In view of the characteristics of future airport construction, such as complex environment, harsh climate, dangerous road, and difficulty in reaching by people and cars, lowaltitude photogrammetry, GNSS and GIS technologies are applied to each key link of airport construction, providing the most direct first-hand data for engineers and technicians to grasp the airport construction situation from a macro perspective. At the same time, it provides a more intuitive and vivid data display form for macro decision-makers and assists design units to further adjust and optimize the design.

\section{Acknowledgements}

This research was funded by the China Civil Aviation Safety Capacity Building Fund Project: Realization and Construction Demonstration of Digital Quality Monitoring System for the Construction of Gravel Pile in Airport Projects.

\section{References}

1. F. Cao, Exploration on Promoting High Quality Development of Geological Surveying and Mapping Units, Economy of Geological Exploration, 8, 5861+67 (2019)

2. R. Li, Discuss the Function and Development Trend of Modern Surveying and Mapping Technology, Jiangxi Building Materials, 5, 14+200 (2019)

3. C. Guo, J. Nie, J. Tian, W. Wang, C. Cheng, B. Wang, H.Yin, H. Zhang, Vertical ground displacements in the Shandong Province derived from long-term GNSS and leveling surveying, Adv. Space. Res, 64:7, 1388-1397 (2019)

4. A.T. Mozas-Calvache, F.J. Ariza-Lopez, Analysing the positional accuracy of GNSS multi-tracks obtained from VGI sources to generate improved 3D mean axes, Int. J. Geogr. Inf. Sci, 33:11, 2170-2187 (2019) 
5. B. Hu, H. Zhang, J. Zhang, Attitude accuracy assessment technique of aircraft inertial navigation system based on low-altitude photogrammetry, China Measurement \& Test, 45, 145-150 (2019)

6. R. Jing, Layout program of image control points in photogrammetry technology based on UAV-Applied in water conservancy and hydropower projects, Journal of East China University of Technology (Natural Science), 4286-90 (2019)

7. S. Mala, M.K. Jat, Geographic information system based spatio-temporal dengue fever cluster analysis and mapping, The Egyptian Journal of Remote Sensing and Space Sciences, (2019)

8. Z. Zhang, G. Hu, F. Wu, C. Wang, The Campus Surveying Control Network Digital Construction Planning and Implementation, 2018 26th International Conference on Geoinformatics, 1-5 (2018)

9. C. Raffaela, J.B. Zielinski, M. Barbarella, New Advanced GNSS and 3D Spatial Techniques, (Springer, 2018)

10. D. Ding, L. Cai, X. Wang, B. Shao, Y. Zheng, Application of Comprehensive Evaluation of the Airport Site Selection, Appl. Mech. Mater, 97-98, 311-315 (2011) 